\title{
Persistent circular currents of exciton-polaritons in cylindrical pillar microcavities
}

\author{
V. A. Lukoshkin, ${ }^{1,2}$ V. K. Kalevich, ${ }^{1,2}$ M. M. Afanasiev, ${ }^{1,2}$ K. V. Kavokin,,${ }^{1,2}$ Z. Hatzopoulos, ${ }^{3}$ P. G. Savvidis, $, 3,5$ \\ E. S. Sedov, ${ }^{6,7}$ and A. V. Kavokin ${ }^{1,6,8}$ \\ ${ }^{1}$ Spin Optics Laboratory, Saint Petersburg State University, 1 Ulianovskaya, St. Petersburg 198504, Russia \\ ${ }^{2}$ Ioffe Institute, Russian Academy of Sciences, 26 Politechnicheskaya, St. Petersburg 194021, Russia \\ ${ }^{3}$ IESL-FORTH, P.O. Box 1527, Heraklion 71110, Greece \\ ${ }^{4}$ Department of Materials Science and Technology, University of Crete, Heraklion 71003, Greece \\ ${ }^{5}$ National Research University for Information Technology, Mechanics and Optics (ITMO), St. Petersburg 197101, Russia \\ ${ }^{6}$ School of Physics and Astronomy, University of Southampton, Highfield, Southampton SO171BJ, United Kingdom \\ ${ }^{7}$ Department of Physics and Applied Mathematics, Vladimir State University named after A.G. and N.G. Stoletovs, \\ 87 Gorky Str., Vladimir 600000, Russia \\ ${ }^{8}$ CNR-SPIN, Viale del Politecnico 1, I-00133 Rome, Italy
}

(Received 23 August 2017; revised manuscript received 20 March 2018; published 25 May 2018)

\begin{abstract}
We have experimentally observed an eddy current of exciton polaritons arising in a cylindrical GaAs/AlGaAs pillar microcavity under the nonresonant optical pumping. The polariton current manifests itself in a MachZehnder interferometry image as a characteristic spiral that occurs due to the interference of the light emitted by an exciton-polariton condensate with a reference spherical wave. We have experimentally observed the condensates with the topological charges $m=+1, m=-1$, and $m=-2$. The interference pattern corresponding to the $m=-2$ current represents the twin spiral emerging from the center of the micropillar. The switching between the current modes with different topological charges is achieved by a weak displacement of the pump spot.
\end{abstract}

DOI: 10.1103/PhysRevB.97.195149

\section{INTRODUCTION}

Exciton-polaritons are superposition quasipartcles formed in the strong exciton-photon coupling regime in various semiconductor structures. Since 1992, a particular attention has been attracted to exciton-polaritons in semiconductor microcavities [1]. Several fascinating effects linked to the bosonic nature of exciton-polaritons have been observed, including, e.g., the stimulated scattering [2], Bose-Einstein condensation, and polariton lasing [3,4]. The formation of bosonic condensates of exciton-polaritons that is at the heart of polariton lasing manifests itself by the spontaneous emission of a coherent and monochromatic light by a microcavity $[5,6]$. Being formed by weakly interacting bosonic quasiparticles, polariton condensates exhibit some characteristic features of quantum fluids including the quantized vortices, similar to those observed in superconductors and in the superfluid helium [7].

The spontaneous formation of vortex-antivortex pairs in nonresonantly pumped polariton condensates has been observed in Refs. [8-10]. The spontaneous generation of persistent circular currents is expected in polariton liquids confined in annular traps [11]. Such currents represent a significant interest from the fundamental point of view, and may be promising for applications in quantum interferometers and gyroscopes. Circular persistent currents are being studied in cold atomic condensates in optical traps generated by Laguerre-Gaussian light modes that transfer their angular momentum to the atomic condensate [12-14]. Transfer of orbital angular momentum to an exciton-polariton condensate by using optically induced chiral polaritonic lenses has been reported in Ref. [15]. Spontaneous formation of vortices in the systems of coupled pillars has been demonstrated in Ref. [16].
In this context, exciton-polariton condensates offer an advantage of relatively easy tailoring of their shapes due to the repulsion of the coherent condensate fraction from the excitonic reservoir generated by a spatially inhomogeneous optical pumping [17,18]. Moreover, chemical etching of planar semiconductor microcavities allows for formation of pillars, providing a deep confining potential for excitons-polaritons [19-21]. The theory of polariton quantum liquids is now well developed [22]. The experimental methods of generation and detection of polariton circular currents are discussed in Refs. [23-25].

In our previous papers, we have demonstrated experimentally the formation of the ring-shaped polariton condensates in cylindrical pillars of different diameters [20,21]. In this paper, we report on the experimental observation of persistent circular polariton currents with different projections of the orbital momentum to the axis of the structure that correspond to different topological charges $m$ in cylindrical semiconductor micropillars under nonresonant optical excitation with Gaussian beams at the center of the pillar.

\section{SAMPLE AND SETUP}

A Bose-Einstein condensate represents a macroscopically occupied single quantum state [26]. Herewith, the multiparticle quantum system can be described by a single complex wave function $\Psi=\Psi(\mathbf{r}, t) \exp [i \Phi(\mathbf{r}, t)]$, possessing a well-defined quantum mechanical phase $\Phi$. Photons, emitted by the condensate, keep information about the condensate phase. This opens the possibility to investigate coherent properties of the excitonpolariton condensate using optical interferometry methods. For 


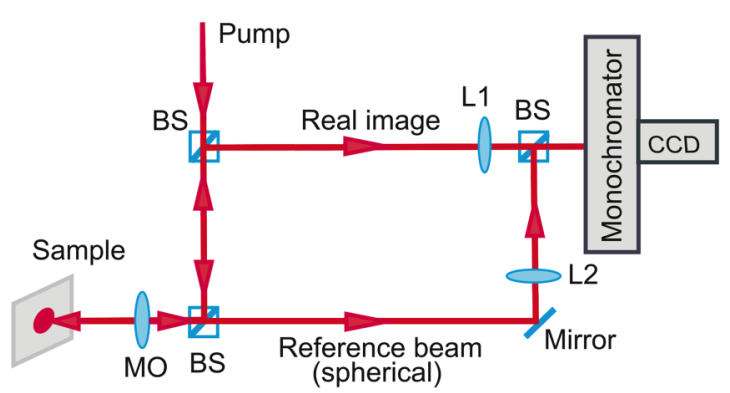

FIG. 1. Schematic of the interferometer setup for the investigation of coherent properties of the exciton-polariton condensate in a micropillar.

this purpose, we use the Mach-Zehnder interferometer with the reference spherical wave.

The general scheme of our setup is presented in Fig. 1. The exciton-polariton condensate in the pillar microcavity was nonresonantly pumped by a cw Ti:sapphire laser tuned to the local minimum of the upper stop-band of the distributed Bragg reflector (about $110 \mathrm{meV}$ above the low polariton branch minimum). Because of the nonresonant excitation, we do not imprint any phase in the condensate directly with the pump laser. The linearly polarized laser beam was focused to a $2 \mu \mathrm{m}$ spot by a microscope objective (MO) of the numerical aperture of 0.42 . The same MO was used to collect the photoluminescence (PL) from the condensate. The parallel PL beam after MO passes through the beamsplitter (BS) and splits into two beams. The upper (in the scheme in Fig. 1) beam, passing through the lens L1 (focal length $F=700 \mathrm{~mm}$ ) produces an enlarged $(\approx 6 \mathrm{~mm})$ real image of the condensate at the entrance slit of the $50-\mathrm{cm}$ monochromator. The lens L2 $(F=100 \mathrm{~mm})$ transforms the lower beam to the spherical wave that is used as a reference. Both beams overlap at the output BS of the interferometer, and their superposition is projected on the entrance slit of the monochromator. The interferogram (or the near-field image of the condensate if the reference beam is blocked) was recorded by the cooled CCD camera at the output of the monochromator.

All experiments were performed at normal incidence of the excitation beam on the sample. A cut-off interference filter was installed in front of the monochromator entrance slit to suppress the excitation laser radiation scattered from the pillar surface. The sample under study was kept in the helium-flow cryostat at $T=3.5 \mathrm{~K}$.

We have examined a set of cylindrical pillars with a diameter of $25 \mu \mathrm{m}$ that were etched from a planar $5 \lambda / 2$ AlGaAs distributed Bragg reflector microcavity with the measured quality factor of $Q=16000$. Four sets of three $10-n m$ GaAs quantum wells are placed at the antinodes of the cavity electric field to maximize the exciton-photon coupling [27]. The microcavity wedge allowed scanning across the sample to set the detuning energy $\delta=E_{C}-E_{X}$, where $E_{C}$ and $E_{X}$ are energies of the cavity mode and of the heavy-hole exciton at zero in-plane wave vector. The studied pillars are characterized by a negative photon-exciton detuning energy $\delta=-(0.5 \div 3.5) \mathrm{meV}$. Within this range, we have found no strong qualitative variation of the observed effects.
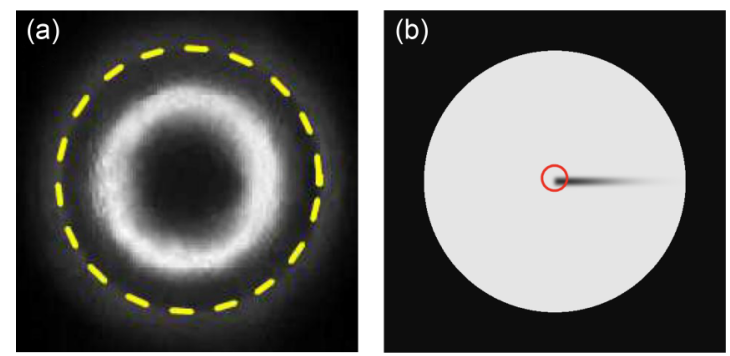

FIG. 2. (a) The real-space image of the exciton-polariton condensate measured in the cylindrical micropillar of a diameter of $25 \mu \mathrm{m}$ under the nonresonant pump in the center of the pillar. The energy of the excitation quanta is $h v_{\mathrm{exc}}=1.664 \mathrm{eV}$, the pump power is $P \approx 1.5 P_{\text {th }}$, the temperature is $T=3.5 \mathrm{~K}$. The dashed circumference shows the edge of the pillar. The plot is normalized to the maximum intensity. (b) Sketch of the potential landscape used for simulations. The stripe inside the pillar shows the assumed defect. The red circle indicates the half maximum intensity of the optical pump spot (its shift from the pillar center is enlarged for clarity).

\section{EXPERIMENTAL RESULTS}

The real-space image of the condensate and its interferogram measured when the pump beam was focused close to the pillar center are shown in Fig. 2(a) and in the upper panel in Fig. 3(a), respectively, for the pump power $P \approx 1.5 P_{\text {th }}$, where $P_{\text {th }}=2.8 \mathrm{~mW}$ is the condensate threshold. As seen in Fig. 2(a), the condensate has the shape of a symmetric ring with the diameter $(\approx 16 \mu \mathrm{m})$ strongly exceeding the size of the excitation $\operatorname{spot}(\approx 2 \mu \mathrm{m})$, in full agreement with our earlier observations $[20,21]$ in what concerns the dependence of the condensate shape on the diameter of the pillar, the intensity, and the position of the pumping beam. The interferogram in the upper panel in Fig. 3(a) has the form of a set of concentric rings, evidencing the absence of the nontrivial topological charge in the condensate, $m=0$. It is worth noting that the nearly perfect shape of the interference rings, as well as their concentricity, certify the sphericity of the wave formed in the reference arm of our interferometer.

A small displacement (less than $1 \mu \mathrm{m}$, see details below) of the pumping spot from the position where the image with $m=0$ in the upper panel in Fig. 3(a) was recorded leads to a dramatic change of the interferogram. In particular, the interference pattern can be transformed into the single spiral turning counter-clockwise [the middle panel in Fig. 3(a)] or clockwise [the lower panel in Fig. 3(a)].

The phases of the condensate emission relative to that of the spherical wave directly extracted from the interferogram images are presented in Fig. 3(b). To extract the relative phase from the interferograms in Fig. 3(a), we use the conventional Fourier-transform method. To overcome the constraints associated with the closed-fringe patterns, we split the interferograms into several overlapping fragments containing only openfringe patterns suitable for implementation of the method. The subsequent splicing of the outcomes gives the phases corresponding to the original closed-fringe interferograms. The spiral shape of the interference fringes and the relative phase patterns indicate that the dependence of the condensate phase $\Phi$ on the azimuth angle $\theta$ is close to linear: $\Phi=m \theta$. 
(a)
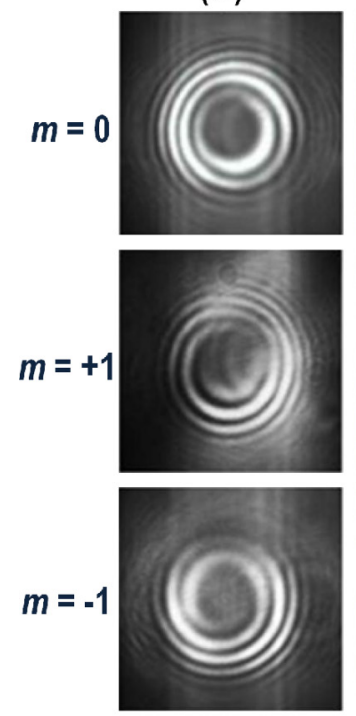

(b)
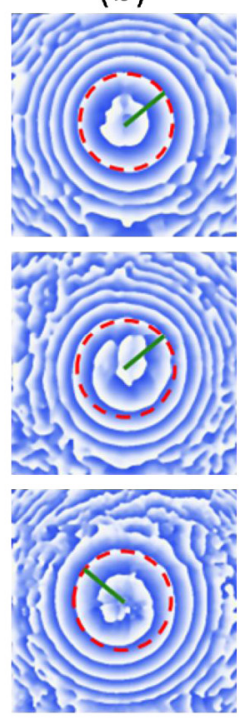

(c)
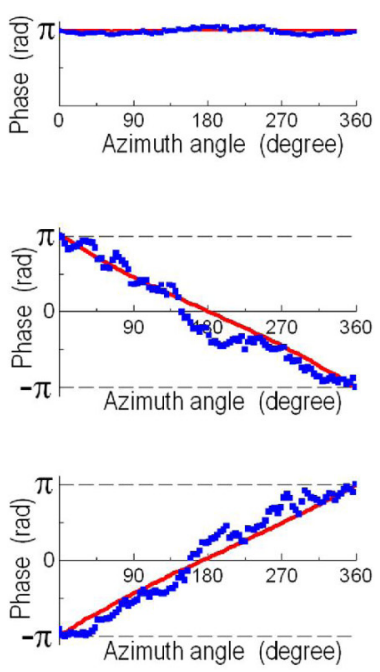

(d)
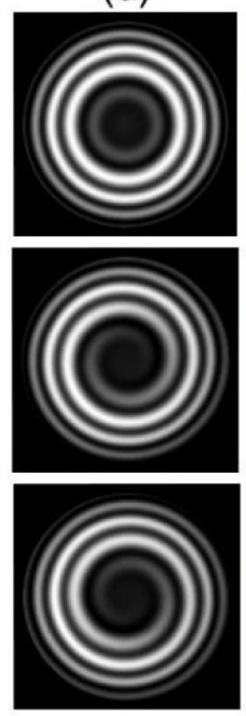

(e)

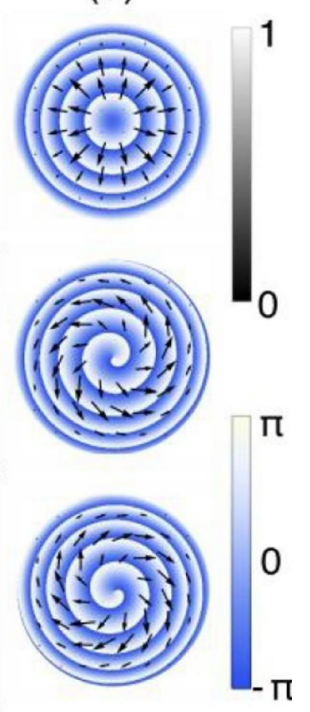

FIG. 3. The experimental interferometry images (a), the relative phase maps extracted from the interferometry images (b), the experimental (solid squares), and calculated (red solid lines) phase variations along the red dashed circumferences in Fig. 3(b), taken counterclockwise from the green radii (c), the simulated interferometry images (d), and relative phases (e) of the exciton-polariton condensates with the topological charges $m=0$ (upper panels), $m=+1$ (middle panels), and $m=-1$ (lower panels). The vector field $\mathbf{J}=\operatorname{Im}\left[\psi^{*} \nabla \psi\right]$ (black arrows) is superimposed on the relative phase maps in the panels (e). The parameters used for the modeling are given in Ref. [28].

The observation of one-thread spirals with opposite helicities evidences occurrence of the nontrivial topological charge of the condensate, equal to unity with both possible signs: $m=+1$ and $m=-1$ in the middle and lower panels in Fig. 3(a). Figure 3(c) demonstrates the condensate phase change along the red dashed circumferences in Fig. 3(b), taken counterclockwise from the green radii. The variation of the phase by $-2 \pi$ and $2 \pi$ is observed for the $m=+1$ and $m=-1$ condensates, respectively. For the nonspiraling condensate with $m=0$, the phase remains constant along the condensate ring. For our ring-shaped condensates, the appearance of a nonzero angular momentum indicates that a circular current of polaritons flows around the ring. We would like to stress that once these current states emerge they are quite stable. The interference images remain unchanged for minutes, that is, 13 orders of magnitude longer that the polariton lifetime (about $10 \mathrm{ps}$ ). This brings us to the conclusion that we observe persistent circular currents of Bose-condensed exciton-polaritons trapped in the cylindrical pillar.

All interferograms in Fig. 3(a) were obtained at the same experimental conditions except for the position of the excitation spot, which was slightly different in different experiments. The shift of the excitation spot was certainly less than $2 \mu \mathrm{m}$, which is the positioning accuracy of our micrometric mechanical $\mathrm{X}-\mathrm{Y}$ translation stage that controls the position of the sample with respect to the excitation beam. In our opinion, the real displacement of the pumping spot away from the pillar center was even much less than $1 \mu \mathrm{m}$. This estimation is supported by the fact that the near-field condensate image, which is extremely sensitive to this displacement [20,21], being measured for each specific interferogram, does not show any changes and coincides with the image in Fig. 2(a). This observation suggests a very strong sensitivity of the condensate current states to the effective potential landscape created by the reservoir of incoherent excitons, by the pillar boundary, and by various inhomogenities and defects in microcavity.

Due to a very short polariton lifetime, the polariton condensate exists as long as the optical excitation is present and disappears soon after switching off the pump. Also, the exciton reservoir is emptied on a nanosecond timescale, typically. As a consequence, the newly created condensate, formed once the pump is switched on again, completely loses the information about its previous state. If the persistent circular current had arisen stochastically, as a result of a chaotic uncertainty of the polariton density near the condensation threshold [11,29,30], then, the subsequent switching on of the excitation light would have created interferential spirals of opposite helicities with equal probabilities. However, the experimentally observed spiral conserves its helicity in every switching off and on of the pump. Since in subsequent switching on of the excitation light, all the experimental parameters remain unchanged, we can conclude that the appearance of circular currents is most likely due to the combined effect of structural inhomogeneities of the microcavity and the microscopic shift of the pump spot from the center of the pillar. The combined effect of a stationary disorder and shifted pump spot is capable of breaking the symmetry between clockwise and anticlockwise polariton flows. This effect is analogous to the one of optically induced chiral polaritonic lenses discussed in Ref. [15]. It is important to note that, taken alone, the shift of the pump spot away from the center of the pillar would not break this symmetry. The stationary potential created by the pillar must not be perfectly cylindrically symmetric, which makes us think of hidden-structure inhomogeneities of the pillar. The nature of these inhomogeneities is unknown. Because of the sharp dependence of the shape of our interferograms on microscopic pumping spot displacements, we believe that most likely the stationary disorder comes from a local defect situated near the 
pillar center, which breaks the axial symmetry of scattering of polaritons sliding down the potential hill formed by hot excitons under the excitation spot. We note that while the mechanism of the vortex formation is similar in the present work and in Ref. [15], we operate with a symmetric Gaussian pump spot, and the switching between the circular current states with different topological charges is achieved simply by shifting the pump spot with respect to the center of the pillar. In contrast, in Ref. [15], polaritons are pumped with six unequal pump beams, and the switching between vortices with different topological charges would imply redesigning the whole pump configuration.

\section{RESULTS OF MODELING AND COMPARISON WITH THE EXPERIMENTAL DATA}

The experimentally observed exciton-polariton condensate steady states can be described by the stationary many-body wave function taken in the form $\Psi(\mathbf{r}, t)=\psi(\mathbf{r}) \exp (-i \mu t)$, where $\mu$ is the chemical potential. The wave function $\psi$ obeys the stationary generalized Gross-Pitaevskii equation

$$
\mu \psi=\left[\widehat{E}_{\text {kin }}+V_{\text {eff }}(\mathbf{r})-i \hbar\left(\gamma_{C}-R n_{R}\right) / 2\right] \psi,
$$

where $\widehat{E}_{\text {kin }}=-\hbar^{2} \nabla^{2} / 2 M$ is the kinetic energy operator, $M$ is the effective mass of polaritons. The stationary effective potential $V_{\text {eff }}(\mathbf{r})$ takes the form $V_{\text {eff }}(\mathbf{r})=U(r)+V(\mathbf{r})+\alpha_{C}|\psi|^{2}+$ $\alpha_{R} n_{R}$. The first term $U(r)$ describes the stationary axially symmetric trapping potential governed by the geometry of the structure. The second term $V(\mathbf{r})$ is responsible for the reduction of cylindrical symmetry of the pillar due to possible defects and inhomogeneities. The two last terms in $V_{\text {eff }}(\mathbf{r})$ describe corrections to the potential landscape due to the intracondensate polariton-polariton interactions and the polariton interactions with the reservoir of hot excitons, respectively. The parameters $\alpha_{C}$ and $\alpha_{R}$ are the corresponding polariton-polariton and polariton-exciton coupling constants. The stationary density of excitons in the reservoir is $n_{R}(\mathbf{r})=P(\mathbf{r}) /\left(\gamma_{R}+R|\psi|^{2}\right)$, where $P(\mathbf{r})$ is the inhomogeneous nonresonant optical pump and $R$ is the stimulated scattering rate describing the particle exchange between the polariton condensate and the exciton reservoir. We consider the azimuthally symmetric Gaussian pump, $P(\mathbf{r})=P_{0} \exp \left[-\left(\mathbf{r}-\mathbf{r}_{s}\right)^{2} / w_{p}^{2}\right]$, shifted by the vector $\mathbf{r}_{s}=\left(x_{s}, y_{s}\right)$ from the center of the pillar. The intensity $P_{0}$, the width of the pump beam $w_{p}$, and the shift $\mathbf{r}_{s}$ are the fitting parameters of the model. The imaginary term in the right-hand side of Eq. (1) is responsible for the balance of the gain from the pumped reservoir and the radiative losses. The factors $\gamma_{C}$ and $\gamma_{R}$ are the loss rates of the condensate polaritons and the reservoir excitons, respectively.

Figure 3(d) shows the results of the numerical simulation of the stationary intensity patterns appearing due to the interference of light emitted by the ring-shaped polariton condensate with the reference spherical wave. The topological charges $m=0,+1$, and -1 correspond to the upper, middle, and lower panels, respectively. The topological charge was calculated as $m=(2 N)^{-1} \int_{S}\left[x\left(J_{y}-\bar{J}_{y}\right)-y\left(J_{x}-\bar{J}_{x}\right)\right] d \mathbf{r}$, where $S$ is the area enclosing the condensate density distribution [23,31], $\mathbf{J}=$ $\operatorname{Im}\left[\psi^{*} \nabla \psi\right]\left(\overline{\mathbf{J}}=\operatorname{Im}\left[\psi \nabla \psi^{*}\right]\right)$ characterizes the polariton flow in the stationary state, and $N$ is the wave function normalization constant describing the population of the condensate. The cor-
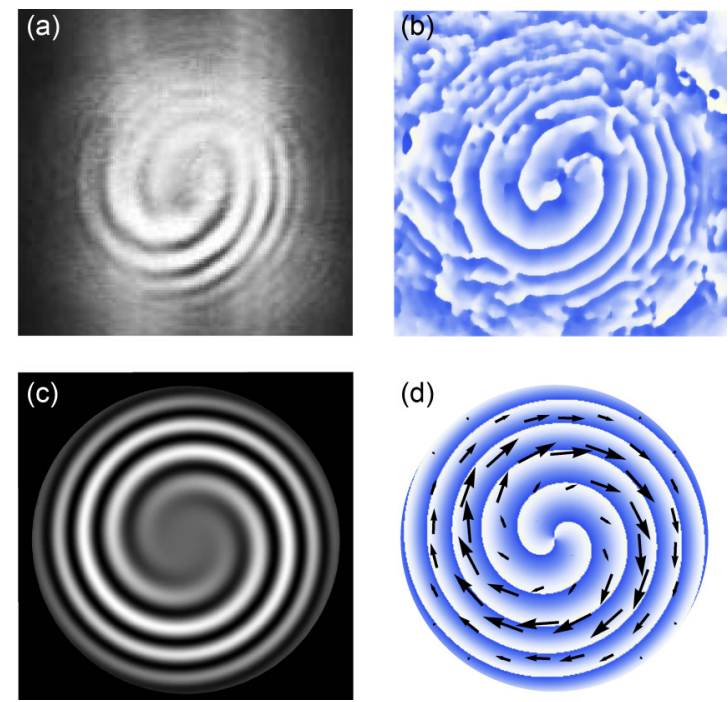

FIG. 4. The experimental interferogram (a) and the relative phase (b) of the exciton-polariton condensate with the topological charge $m=-2$ recorded in the $25 \mu \mathrm{m}$ cylindrical pillar. The modelled interferogram (c) and relative phase (d) of the $m=-2$ condensate. Arrows in map (d) show the direction and the magnitude of the flow $\mathbf{J}$ of polaritons. The color scale in panels (b) and (d) is the same as in Figs. 3(b) and 3(e).

responding spatial variation of the relative phase provided with the vector plot of the field $\mathbf{J}$ is presented in Fig. 3(e). The vector field $\mathbf{J}$ winds around the vortex core. The spiraling intensity fringes in the interference pattern evidence the presence of the circular current of polaritons in the ring condensate. In the model, we consider the pillar with a small elongated defect situated near its center and oriented along the radius of the pillar. Potential landscape used for modeling is schematically presented in Fig. 2(b). If the pump spot is shifted from the center of the pillar by the tiny submicrometer distances, the condensate vortex states are still supported. However, in the case of the pump spot shifted clockwise (anticlockwise) with respect to the defect axis, only the clockwise (anticlockwise) spiraling condensates can appear.

While persistent currents with $m= \pm 1$ are relatively easy to obtain, higher topological charges can scarcely be seen. So far, we managed to experimentally realize only the state with $m=$ -2 . The corresponding interferogram and the extracted relative phase given in Figs. 4(a) and 4(b), respectively, represent twin spirals emerging from the pillar center clockwise. The twinspiral state, once obtained, also demonstrates a high stability, persisting for a few minutes. The corresponding stationary interference pattern and the spatial distribution of the relative phase for the $m=-2$ state, obtained from a numerical solution of Eq. (1), are shown in Figs. 4(c) and 4(d), respectively.

\section{CONCLUSIONS}

We have demonstrated experimentally the existence of persistent circular currents in the ring-shaped exciton-polariton condensates appearing in cylindrical micropillars under the nonresonant optical pumping. We have observed the quantum states of ring-shaped condensates characterized by topological 
charges $m= \pm 1,-2$. The polariton currents are sustained by the balance of the gain due to the optical pump and the loss due to the finite polariton lifetime. Once established, the current is preserved by the condensate within the time duration of the experiment. Its topological charge would not change at switching the optical pumping off and on. This observation indicates that the symmetry breaking between clockwise and anticlockwise polariton flows is a result of the interplay of an asymmetric stationary disorder potential and the microscopic shift of the pump spot from the center of the pillar. In spite of the yet-unknown nature of the disorder imperfections leading to the vorticity, cylindrical micropillars proved to be convenient objects for realization of circular polariton currents. The observation of persistent circular currents of exciton-polaritons paves the way to multiple applications of polariton condensates in quantum interference devices [32-35].

\section{ACKNOWLEDGMENTS}

The work of V.A.L., V.K.K., M.M.A., K.V.K., and A.V.K. was supported in part by the RFBR (Grant No. 15-52-12018) within the joint Russian-German Project No. ICRC TRR160 and by Saint Petersburg State University via the research Grant No. 11.34.2.2012. P.G.S. acknowledges bilateral GreeceRussia Polisimulator Project co-financed by Greece and the EU Regional Development Fund. E.S.S. thanks the RFBR Grants No. 16-32-60104 and No. 17-52-10006. A.V.K. acknowledges the support from the EPSRC Programme Grant on Hybrid Polaritonics No. EP/M025330/1 and the partial support from the HORIZON 2020 RISE project CoExAn (Grant No. 644076). E.S.S. and A.V.K. acknowledge the support from the Royal Society International Exchange Grant No. IEC/R2/170227.
[1] C. Weisbuch, M. Nishioka, A. Ishikawa, and Y. Arakawa, Phys. Rev. Lett. 69, 3314 (1992).

[2] P. G. Savvidis, J. J. Baumberg, R. M. Stevenson, M. S. Skolnick, D. M. Whittaker, and J. S. Roberts, Phys. Rev. Lett. 84, 1547 (2000).

[3] J. Kasprzak, M. Richard, S. Kundermann, A. Baas, P. Jeambrun, J. M. J. Keeling, F. M. Marchetti, M. H. Szymańska, R. André, J. L. Staehli, V. Savona, P. B. Littlewood, B. Deveaud, and L. S. Dang, Nature 443, 409 (2006).

[4] R. Balili, V. Hartwell, D. Snoke, L. Pfeiffer, and K. West, Science 316, 1007 (2007).

[5] A. Imamoglu, R. J. Ram, S. Pau, and Y. Yamamoto, Phys. Rev. A 53, 4250 (1996).

[6] S. Christopoulos, G. B. H. von Hogersthal, A. J. D. Grundy, P. G. Lagoudakis, A. V. Kavokin, J. J. Baumberg, G. Christmann, R. Butte, E. Feltin, J.-F. Carlin, and N. Grandjean, Phys. Rev. Lett. 98, 126405 (2007).

[7] A. J. Laggett, Quantum Liquids (Oxford University Press, Oxford, 2008).

[8] K. G. Lagoudakis, M. Wouters, M. Richard, A. Baas, I. Carusotto, R. André, L. S. Dang, and B. Deveaud-Plédran, Nat. Phys. 4, 706 (2008).

[9] G. Nardin, G. Grosso, Y. Léger, B. Piétka, F. Morier-Genoud, and B. Deveaud-Plédran, Nat. Phys. 7, 635 (2011).

[10] G. Roumpos, M. D. Fraser, A. Löffler, S. Höfling, A. Forchel, and Y. Yamamoto, Nat. Phys. 7, 129 (2011).

[11] A. V. Nalitov, T. C. H. Liew, A. V. Kavokin, B. L. Altshuler, and Y. G. Rubo, Phys. Rev. Lett. 119, 067406 (2017).

[12] A. Ramanathan, K. C. Wright, S. R. Muniz, M. Zelan, W. T. Hill, C. J. Lobb, K. Helmerson, W. D. Phillips, and G. K. Campbell, Phys. Rev. Lett. 106, 130401 (2011).

[13] S. Moulder, S. Beattie, R. P. Smith, N. Tammuz, and Z. Hadzibabic, Phys. Rev. A 86, 013629 (2012).

[14] K. C. Wright, R. B. Blakestad, C. J. Lobb, W. D. Phillips, and G. K. Campbell, Phys. Rev. Lett. 110, 025302 (2013).

[15] R. Dall, M. D. Fraser, A. S. Desyatnikov, G. Li, S. Brodbeck, M. Kamp, C. Schneider, S. Höfling, and E. A. Ostrovskaya, Phys. Rev. Lett. 113, 200404 (2014).
[16] V. G. Sala, D. D. Solnyshkov, I. Carusotto, T. Jacqmin, A. Lemaître, H. Terças, A. Nalitov, M. Abbarchi, E. Galopin, I. Sagnes, J. Bloch, G. Malpuech, and A. Amo, Phys. Rev. X 5, 011034 (2015).

[17] G. Tosi, G. Christmann, N. G. Berloff, P. Tsotsis, T. Gao, Z. Hatzopoulos, P. G. Savvidis, and J. J. Baumberg, Nat. Phys. 8, 190 (2012).

[18] Y. Sun, Y. Yoon, S. Khan, L. Ge, M. Steger, L. N. Pfeiffer, K. West, H. E. Türeci, D. W. Snoke, and K. A. Nelson, Phys. Rev. B 97, 045303 (2018).

[19] D. Bajoni, P. Senellart, E. Wertz, I. Sagnes, A. Miard, A Lemaître, and J. Bloch, Phys. Rev. Lett. 100, 047401 (2008).

[20] V. K. Kalevich, M. M. Afanasiev, V. A. Lukoshkin, K. V. Kavokin, S. I. Tsintzos, P. G. Savvidis, and A. V. Kavokin, J. Appl. Phys. 115, 094304 (2014).

[21] V. K. Kalevich, M. M. Afanasiev, V. A. Lukoshkin, D. D. Solnyshkov, G. Malpuech, K. V. Kavokin, S. I. Tsintzos, Z. Hatzopoulos, P. G. Savvidis, and A. V. Kavokin, Phys. Rev. B 91, 045305 (2015).

[22] J. Keeling and N. G. Berloff, Contemp. Phys. 52, 131 (2011).

[23] G. Li, M. D. Fraser, A. Yakimenko, and E. A. Ostrovskaya, Phys. Rev. B 91, 184518 (2015).

[24] X. Ma, U. Peschel, and O. A. Egorov, Phys. Rev. B 93, 035315 (2016).

[25] G. Liu, D. W. Snoke, A. Daley, L. N. Pfeiffer, and K. West, Proc. Natl. Acad. Sci. USA 112, 2676 (2015).

[26] C. N. Yang, Rev. Mod. Phys. 34, 694 (1962).

[27] P. Tsotsis, P. S. Eldridge, T. Gao, S. I. Tsintzos, Z. Hatzopoulos, and P. G. Savvidis, New J. Phys. 14, 023060 (2012).

[28] In the modeling, we take the following values of the parameters: the effective polariton mass is $M=5 \cdot 10^{-5} m_{e}$ with $m_{e}$ being a free electron mass, the decay rates are $\gamma_{C}=0.033 \mathrm{ps}^{-1}$ and $\gamma_{R}=0.5 \mathrm{ps}^{-1}$, the interaction constants are $\alpha_{C}=4 \mathrm{meV} \mu \mathrm{m}^{2}$ and $\alpha_{R}=2 \alpha_{C}$, the condensate-reservoir scattering rate is $R=0.06 \mathrm{ps}^{-1} \mu \mathrm{m}^{2}$.

[29] H. Ohadi, A. Dreismann, Y. G. Rubo, F. Pinsker, Y. del ValleInclan Redondo, S. I. Tsintzos, Z. Hatzopoulos, P. G. Savvidis, and J. J. Baumberg, Phys. Rev. X 5, 031002 (2015). 
[30] A. V. Yulin, A. S. Desyatnikov, and E. A. Ostrovskaya, Phys. Rev. B 94, 134310 (2016).

[31] M. S. Soskin, V. N. Gorshkov, M. V. Vasnetsov, J. T. Malos, and N. R. Heckenberg, Phys. Rev. A 56, 4064 (1997).

[32] I. A. Shelykh, G. Pavlovic, D. D. Solnyshkov, and G. Malpuech, Phys. Rev. Lett. 102, 046407 (2009).
[33] G. Franchetti, N. G. Berloff, and J. J. Baumberg, arXiv:1210.1187v2.

[34] C. Ryu, P. W. Blackburn, A. A. Blinova, and M. G. Boshier, Phys. Rev. Lett. 111, 205301 (2013).

[35] F. I. Moxley, J. P. Dowling, W. Dai, and T. Byrnes, Phys. Rev. A 93, 053603 (2016). 